\title{
Percepción del acoso sexual según los niveles de prejuicio sexista
}

\author{
Perception of sexual harassment according to sexist prejudice levels
}

\author{
Anahí Mailén López¹, Talía Gómez Yepes²
}

\begin{abstract}
RESUMEN
El prejuicio sexista en sus formas hostil o benevolente favorece la estereotipia de la mujer. Asimismo, de acuerdo a la teoría del rol social, las personas están circunscritas a tareas determinadas porque existen disposiciones inherentes a ellas que generan tal correspondencia; en consecuencia, se infiere que hombres y mujeres se adscriben a determinados roles sociales tradicionales por sus cualidades personales, evaluación que deriva en los estereotipos de género. El objetivo principal de este trabajo fue analizar las percepciones sobre el acoso sexual por parte de hombres y mujeres, así como su relación con el sexismo ambivalente (en su forma hostil como benevolente) y la ideología de los roles sociales de género. Para tal fin, se trabajó con una muestra de 432 participantes (mujeres, 64.54\%; hombres, 35.46\%) con una media de 31.09 años, residentes en el Área Metropolitana de Buenos Aires. Los resultados indican un amplio porcentaje de invisibilización del acoso sexual, siendo los hombres quienes lo perciben significativamente menos que las mujeres. Asimismo, se hallaron diferencias en la percepción del acoso sexual según los niveles de sexismo hostil y benevolente, así como también con respecto a la ideología del rol de género. Se discuten los resultados en contraste con datos obtenidos en estudios previos sobre el acoso sexual, así como las relaciones con variables psicosociales.
\end{abstract}

Palabras clave: Acoso sexual, Sexismo, Género, Prejuicio.

\begin{abstract}
Sexist prejudice in its hostile or benevolent forms helps the women's stereotypy. Besides, according to Social Role theory, people are circumscribed to specific tasks, owing to the fact that there are inner dispositions which generate those correspondences. As a result, it is inferred that men and women assign themselves certain sex-typical social role because of their personal qualities. This evaluation results in gender stereotypes. We aimed to analyze men and women's perceptions about sexual harassment, and the relationship between ambivalent sexism (in benevolent and sexism forms), gender roles and participant sex. Participants were 432 (women, 64.54\% ; men, 35.46\%) citizens from Ciudad Autónoma de Buenos Aires. The mean age of respondents was 31.9 years. Findings provide strong support for our overall predictions about invisibility of sexual harassment. These suggest that men perceive significantly less harassment than women. But also, ambivalent sexism and genre roles perception differences were found. Moreover, these findings are discussed in contrast to previous research data about sexual harassment and its relation to psychosocial variables.
\end{abstract}

Keywords: Sexual harassment, Sexism, Gender, Prejudice. 


\section{Introducción}

A pesar de los esfuerzos llevados a cabo desde diferentes organismos internacionales (CEDAW, 1979; OEA, 1994; ONU, 1995; European Union Agency for Fundamental Rights, 2014) las mujeres continúan siendo víctimas de diferentes formas de violencia y discriminación. La violencia hacia niñas, adolescentes y mujeres es la violación de derechos humanos más frecuente, intensa y transversal, dado que está presente en todos los países, culturas y clases sociales (Amnistía Internacional, 2017; Guedes, García-Moreno \& Bott, 2014; Expósito \& Moya, 2005). Según la Organización Mundial de la Salud (2013) el $35 \%$ de las mujeres en el mundo experimentó hechos de violencia (en la pareja o fuera de ella) en algún momento de sus vidas. Por su parte, la Oficina de Objetivos de Desarrollo Sostenible de la Comisión Económica para América Latina y el Caribe (CEPAL) informó que sobre la base de datos de 2005 a 2016 para 87 países, el 19\% de las mujeres de entre 15 y 49 años dijeron haber sufrido violencia física y/o sexual por parte de su pareja en los 12 meses previos a la encuesta y debido a la universalidad de este fenómeno, la Agenda 2030 para el Desarrollo Sostenible (CEPAL, 2018) tiene por objetivo eliminar todas las formas de violencia contra las mujeres y las niñas en los ámbitos público y privado.

Argentina no está exenta de esta problemática. Las tasas de violencia de género siguen siendo preocupantemente altas, el acceso a los recursos productivos continúa siendo desigual y la brecha salarial de género sumamente amplia (OECD, 2014). A pesar de la sanción de la Ley Nacional 26.485/2009 de Protección Integral para Prevenir, Sancionar y Erradicar la Violencia contra las Mujeres, los datos estadísticos demuestran que es un fenómeno constante en la sociedad actual; según el primer informe oficial, el Registro de Casos de Violencia Contra las Mujeres (INAM, 2017; INDEC, 2018; Defensoría del Pueblo, 2017) en el país una de cada diez mujeres ha sido víctima de algún tipo de violencia. Entre 2013 y 2017 se registraron un total de 260.156 casos de violencia contra las mujeres, con un predominio de la violencia psicológica $(86.9 \%$ de los casos), seguida de la física $(67.4 \%)$, la simbólica (25.1\%), la económica y patrimonial (19.4\%) y la sexual $(7.9 \%)$. Sin embargo, estas cifras no constituyen el universo total de las mujeres que padecen violencia, debido a que no todas se atreven a denunciar estas situaciones (INDEC, 2018). Por su parte, la Oficina de Violencia Doméstica de la Corte Suprema de Justicia de la Nación Argentina, en su informe del primer trimestre de 2018, reveló que el $78 \%$ de los individuos denunciados por hechos de violencia son del sexo masculino. Los varones entre 22 y 49 años representan el $63 \%$ de las personas denunciadas (OVD, 2018).

Una de las modalidades más frecuentes de la violencia hacia niñas, adolescentes y mujeres es el acoso sexual. En sus diversas formas, éste constituye un fenómeno social generalizado que afecta a las personas tanto en el trabajo como en contextos sociales informales (Galdi, Mass y Cadinu, 2013; Stockdale, 1998; Stockdale, Gandolfo,
Schneider \& Cao, 2004). Si bien el término "acoso sexual" define una modalidad de acoso que puede afectar a hombres y a mujeres (Moyer \& Nath, 1998), el mayor número de víctimas de acoso sexual en el ámbito de trabajo son mujeres (OIT, 2013, 2017).

El término acoso sexual incluye lo que en Ciencias Sociales se denomina "acoso de género", es decir, aquellos comportamientos hostiles, insultos y/o actos degradantes que sirven para expresar actitudes insultantes u ofensivas hacia las mujeres (Expósito \& Moya, 2005; Holland, Rabelo, Gustafson, Seabrook \& Cortina, 2016). Además, el término también incluye la "atención sexual no deseada”, haciendo referencia a aquellos actos sexuales ofensivos no deseados y no recíprocos, que abarca conductas verbales como expresiones, chistes y comentarios de doble sentido (Diehl, Rees \& Bohner, 2016) y no verbales tales como la mirada objetivante (examinar las partes del cuerpo de otra persona), tocarse indebidamente o presionar a alguien para obtener citas (Expósito y Moya, 2005; Galdi et al., 2013). En definitiva, bajo la categoría "acoso sexual" se agrupan conductas que incluyen gestos, expresiones, palabras, contacto físico indebido y no consentido con connotación sexual. Aunque con diferentes gravedades, todas estas conductas constituyen acciones que atentan contra la seguridad física y psicológica de las mujeres, generando un impacto negativo en su salud (Diehl et al., 2016; OMS, 2013; Ramírez, 2016).

Respecto de la percepción de este fenómeno en el ámbito callejero, estadísticas recientes de la Ciudad Autónoma de Buenos Aires llevadas a cabo por el Observatorio de Opinión Pública (UAI, 2018), demuestran que el $85 \%$ de las mujeres encuestadas sufrió alguna situación de acoso sexual callejero. De acuerdo con datos del mismo Observatorio, en el caso de los varones participantes, el $62 \%$ cree que a las mujeres les gustan los piropos callejeros (UAI, 2018). Estos indicadores generan un interrogante acerca de si existe un sesgo de género al momento de percibir el fenómeno y si, como sostienen diferentes estudios psicológicos (Diehl et al., 2016; Fiske, 2018; Russell \& Trigg, 2004; Glick \& Fiske, 1995) el acoso sexual en sus diferentes facetas formaría parte de una dimensión más amplia y general de actitudes hostiles hacia las mujeres. En este sentido, tanto el prejuicio sexista como el sostenimiento de una ideología tradicional de roles de género, constituirían las bases que provocan o desinhiben el acoso (Fiske, 2018; Russell \& Oswald, 2016).

\section{Acoso y prejuicio sexista}

El prejuicio sexista ha sido estudiado como la hostilidad hacia las mujeres por una gran parte de la literatura académica sobre el tema (Fiske, 2018). En la década de los noventa, Glick y Fiske (1996, 2001) plantearon su propuesta sobre el sexismo ambivalente, introduciendo en el plano de la discusión una particularidad que no había sido tenida en cuenta para analizar el fenómeno; lo que sus investigaciones revelaron sobre la naturaleza del prejuicio sexista cuestionaba las definiciones existentes 
hasta el momento acerca del prejuicio como una antipatía hacia un grupo particular (Allport, 1954) y llamaban la atención sobre cómo la tendencia a sobredimensionar la emocionalidad de las mujeres constituye una forma subjetiva de sostener los prejuicios paternalistas y benevolentes que refuerzan la desigualdad social entre hombres y mujeres (Etchezahar, 2014; Gaertner \& Dovidio, 2014). De acuerdo a la teoría del sexismo ambivalente, en un mismo fenómeno convergen dos manifestaciones de significados independientes, pero relacionadas; un componente benevolente y uno hostil, cada uno de los cuales incorpora actitudes relacionadas con los factores estructurales que afectan las relaciones entre hombres y mujeres. Estos componentes funcionan como formas de justificación del poder estructural de los hombres sosteniendo la desventaja de las mujeres (Reyes Aguinaga, 1998), las cuales se presentan en una variedad de culturas como sistemas de creencias coherentes (Glick \& Fiske, 1996; 2001). Si la intención general que conlleva el prejuicio es posicionar en desventaja a un determinado grupo social, entonces, como señalan Gaertner \& Dovidio (2014), no es la antipatía sino la desigualdad social lo que lo motiva. De esta manera, las formas condescendientes y subjetivamente positivas hacia las mujeres (componente benevolente del sexismo), no sólo refuerzan las desigualdades de género, sino que también constituyen una forma de prejuicio (Glick \& Fiske, 2001).

En tal sentido, el sexismo benevolente se define como un conjunto de actitudes que son subjetivamente condescendientes y que, por ende, se perciben como positivas; no solamente por quien las ejecuta sino también por quien las recibe, pero que desde la psicología son consideradas componentes del prejuicio sexista por generar y multiplicar estereotipos negativos hacia las mujeres (Fiske, 2018). Tal estereotipación restringe el espectro de desarrollo de las mujeres, caracterizándolas con atributos de pureza, fragilidad o sostén; y por eso su amor y presencia son necesarios para hacer de los hombres seres completos (Glick \& Fiske, 1996, 2001). Estas actitudes han sido interpretadas históricamente como actos de amor y respeto más que como un acto restrictivo; sin embargo, han ido profundizando y fortaleciendo las brechas entre hombres y mujeres diferenciando los roles de género (Durante \& Fiske, 2017; Eagly, 1987; Eagly, Wood \& Diekman, 2000). Ellas confinadas a un pedestal y al cuidado del hogar, mientras los hombres son percibidos como caballerosos, fuertes, poderosos, protectores y dignos de ocupar espacios de poder (Reyes Aguinaga, 1998).

Contrariamente, el sexismo hostil enmarca un conjunto de actitudes hacia las mujeres que pueden llegar a ser muy violentas y despectivas (Glick \& Fiske, 1996, 2001). El mismo responde al modelo estándar de prejuicio (Allport, 1954) con su definición clásica de "antipatía basada en una generalización inflexible y errónea, que puede ser sentida o expresada y que está dirigida hacia un grupo como totalidad o hacia un individuo por ser miembro de un grupo" (p. 9), aunque en la particularidad del prejuicio sexista presenta un punto de vista contradictorio de confrontación en las relaciones de género, desde el cual los hombres perciben en las mujeres la intención de controlarlos, a través de la sexualidad o de cierta ideología feminista (Glick \& Fiske, 2001). En esta línea, el sexismo hostil es provocado por mujeres que son vistas como un desafío directo o como usurpadoras del poder de los hombres (por ejemplo, feministas, mujeres profesionales, o mujeres seductoras) lo que lo justifica como una "ideología legitimadora" (Pratto, Sidanius \& Stallworth, 1993).

Diferentes investigadores han sostenido que el acoso sexual y otros comportamientos coercitivos podrían hacer parte de una dimensión más amplia y más general de hostilidad hacia las mujeres (Diehl et al., 2016; Fiske, 2018; Russell \& Oswald, 2016; Russell \& Trigg, 2004; Glick \& Fiske, 1995, 1996; Jackman, 1999; Pryor, 1987). Estudios previos (Pryor, Giedd \& Williams, 1995; Pryor \& Whalen, 1997) obtuvieron como resultado que las actitudes hostiles hacia las mujeres estaban relacionadas con la inclinación o propensión al acoso sexual. Ésta propone que, en la medida en que los comportamientos de acoso son aceptados, los hombres que adoptan opiniones sexistas o que no consideran a las mujeres como parte del endogrupo, tienen más probabilidades de albergar actitudes hostiles y manifestarlas en el lugar de trabajo. Un ejemplo de ello es la hostilidad reiterada que los hombres pueden mostrar hacia las mujeres, sin importar si ostentan un cargo de menor o de mayor responsabilidad. Para Glick y Hilt (2000) y Glick y Fiske (1995) el mensaje del acosador es que el rol de la mujer es principalmente sexual y subordinado, no un igual ni en el trabajo, ni en la calle, ni en un mundo de hombres. Sumado a los estudios acerca del sexismo, las investigaciones realizadas desde la Psicología sobre acoso sexual también han identificado que el sostenimiento de una ideología tradicional de los roles sociales de género constituiría una de las variables psicosociales que permitirían la desinhibición y sostenimiento del fenómeno del acoso (Fiske, 2018; Eagly \& Karau, 2002; Russell \& Oswald, 2016).

\section{Rol Social de Género y Acoso sexual}

Históricamente y con el fin de garantizar el sostenimiento económico y los recursos disponibles, las sociedades se han organizado a través de la división de tareas (Geis, 1993) y la reglamentación de estas (Eagly, 1987). Sobre la base de esas leyes se estructura el sistema de normas sociales que abarca, entre otras, las responsabilidades y roles de los miembros de dicha sociedad, conformando así uno de los pilares básicos de la estructura social al establecer y regular las diversas relaciones intergrupales (Eagly \& Wood, 1982) las cuales serán la base de las desigualdades sociales, al tener que ocupar cada individuo una posición diferente en la sociedad (Durante \& Fiske, 2017; Eagly \& Karau, 2002).

De acuerdo con Deaux y Martin (2003) la teoría del Rol Social de Género plantea que los roles son asignados en función del trabajo, ya que cada puesto tiene asociadas una serie de demandas necesarias para su desempeño; de 
esto se infiere que, si una tarea es habitualmente desarrollada por hombres, es que son los hombres los que poseen las capacidades para desarrollarlas. Tales deducciones a su vez se transfieren a otras dinámicas de asignación de roles y expectativas con respecto a ellos mismos (Eagly \& Mladinic, 1993). Los roles sociales y las expectativas de alcanzarlos forman parte de los valores culturales e influyen en el comportamiento de las personas (Geis, 1993). Éstos y las desigualdades que acarrean se perpetúan en la medida en que son aceptados por los individuos, fortaleciendo de manera directamente proporcional la estructura social e ideológica. De acuerdo con Escartí, Musitu y Gracia (1988) será esta consolidación de los roles sociales la que defina la identidad de género, al ser los determinantes de las formas de participación y las tareas que la mayoría de los integrantes de la sociedad desarrollen durante el transcurso de sus vidas.

La Teoría del Rol Social de Género (Eagly, 1987) y la Teoría del Sexismo Ambivalente (Fiske, 2018; Glick \& Fiske, 1996, 2001) permiten abordar el estudio de las relaciones intergrupales (Deaux \& Martín, 2003). A partir de ellas, diferentes autores han sugerido que variables psicosociales como los estereotipos de los roles sociales de género (Fiske, 2018; McCord, Joseph, Dhanani \& Beus, 2018, Russell \& Oswald, 2016) y el prejuicio sexista en sus formas hostil y benevolente, juegan un papel importante en la inclinación y manifestación de violencia y acoso sexual hacia las mujeres (Fisk 2018; Pratto et al. 1993); Pryor, 1987; Glick \& Fiske, 1995, 1996; Jackman, 1999; Glick \& Hilt, 2000), siendo los hombres que creían tener un alto nivel de masculinidad, los más propensos a acosar sexualmente a las mujeres (Pryor, 1987). De acuerdo a Stockdale et al. (2004) la tolerancia al acoso sexual y el respaldo de los roles de género tradicionales están relacionados con la tendencia a malinterpretar la amabilidad de la mujer como un comportamiento sexual, mientras el sentido de masculinidad tradicional también fue asociado con actitudes negativas hacia la igualdad de género, actitudes de apoyo hacia los roles de género tradicionales y las conductas de acoso sexual en espacios de trabajo (Glick \& Fiske, 1995; Pryor, 1987; Stockdale, 1998; Stockdale et al., 2004).

A partir de lo expuesto, el objetivo del presente estudio fue describir las percepciones sobre el acoso sexual de hombres y mujeres, así como analizar si las mismas se diferencian de acuerdo con los niveles de sexismo ambivalente (hostil y benevolente) y la ideología de los roles de género presentes en los participantes de este estudio.

\section{Método}

\section{Diseño}

La presente investigación tuvo un diseño ex post facto prospectivo de comparación de grupos. En este trabajo, se dividió a los participantes en cuatro grupos, según su percepción acerca del acoso sexual, para luego analizar si había diferencias según los niveles de sexismo ambivalente e ideología del rol de género.

\section{Participantes}

Se seleccionó una muestra no probabilística, de tipo incidental, la cual estuvo conformada por 432 adultos, de los cuales 150 eran hombres $(35,46 \%)$ y 273 mujeres $(64,54 \%)$ de entre 18 y 65 años $(M=31,09 ; D T=9,04)$, procedentes del Área Metropolitana de Buenos Aires (AMBA, Argentina).

\section{Instrumento}

El instrumento de recolección de datos consistió en una evaluación autoadministrable, en formato papel y en formato digital, respetando el anonimato de los participantes. La misma estuvo compuesta por las siguientes evaluaciones:

- Percepción de acoso sexual. Se indagó en los participantes a partir de la pregunta " ¿Viviste vos o alguien cercano a vos, alguna situación de acoso sexual?", cuyas respuestas posibles eran: "Nunca”, "Alguna vez", "Algunas veces” y "Muchas veces”.

- Lugares donde vivió situaciones de acoso. A quienes respondieron a la pregunta anterior con las opciones "Alguna vez", Algunas veces" y "Muchas veces", se les solicitó que también indiquen el ámbito en el que se llevó a cabo el acoso sexual, utilizando un formato de respuesta abierta.

- Sexismo Ambivalente. Se utilizó una adaptación local (Etchezahar, 2012) del Inventario de Sexismo Ambivalente original (Glick \& Fiske, 1996, 2001), que evalúa la dimensión Sexismo Hostil como la dimensión Sexismo Benevolente. La evaluación está compuesta por 22 ítems (11 ítems para cada tipo de sexismo), los cuales cuentan con un formato de respuesta tipo Likert con cinco anclajes que varían de forma gradual, desde 1 $=$ "Totalmente en desacuerdo" a 5 = "Totalmente de acuerdo". Tanto la dimensión de sexismo hostil ( $\alpha=$ .81) como la de sexismo benevolente $(\alpha=.79)$ dieron cuenta de confiabilidad y validez para su estudio en la muestra con la que se trabajó.

- Ideología del Rol de Género. Para evaluar este constructo, se utilizó la versión local (Ungaretti \& Etchezahar, 2013) de la Escala de Ideología de Género, cuestionario autoadministrable unidimensional compuesto por 12 ítems. El formato de respuesta de la evaluación es tipo Likert, con anclajes que varían de forma gradual desde 1 = "Totalmente en desacuerdo" a 5 = "Totalmente de acuerdo". Se observaron indicadores de consistencia interna $(\alpha=.84)$ y validez adecuados.

- Variables socio-demográficas. Se indagó por el género y la edad de los participantes.

\section{Procedimiento}

Los participantes fueron invitados a formar parte de este estudio de forma voluntaria, a quienes se le indicó que los datos recolectados eran anónimos y con una finalidad únicamente académico-científica. La recolección se llevó a cabo durante el primer semestre de 2018. La mitad de los respondidos en zonas de conveniencia de la Ciudad de Buenos Aires y el Conurbano Bonaerense, a través de una prueba de lápiz y papel. La otra mitad de 
los participantes lo hicieron a través del Google Forms, respondiendo el mismo instrumento de evaluación que completaron los participantes en la modalidad presencial, pero en formato digital. Para ésta, ambas muestras, se cotejó que los participantes cumplieran con el criterio de residencia indicado previamente. Asimismo, se procedió a analizar una serie de pruebas t con el objetivo de contrastar si los puntajes en las variables estructuradas (escala de Sexismo Ambivalente y escala de Ideología del Rol de Género) variaban entre ambas muestras, no hallando diferencias estadísticamente significativas en ningún caso. Los resultados obtenidos en este estudio fueron procesados con el paquete estadístico SPSS 20.

\section{Resultados}

En primer lugar, se analizaron las frecuencias de la pregunta " $i$ Viviste vos o alguien cercano a vos, alguna situación de acoso sexual?" 148 personas (35.7\%) respondió "nunca"; de los cuales 73 son hombres y 72 mujeres; 3 personas que dieron esta respuesta no especificaron su género. Los participantes restantes, 267 hombres y mujeres, reconocen haber vivido una situación de este tipo, aunque con diferentes frecuencias: $26.5 \%$ respondió “alguna vez"; $22.7 \%$ "algunas veces”; $15.2 \%$ respondió "muchas veces”. Al analizar si había diferencias según género en la percepción de acoso sexual $\left(t_{(405)}=-4.318\right.$; $p<.001 ; d$ de Cohen $=.45$ ), se observó que las mujeres $(M=2.34 ; D T=1.066)$ podrían llegar a percibir más situaciones de acoso sexual con respecto a los hombres $(M=1.87 ; D T=1.029)$.

A continuación, se estudiaron los lugares donde han sufrido o conocen a alguien que haya sufrido este tipo de situaciones: hombres y mujeres coinciden en que el espacio público es el lugar donde más se sufre acoso sexual (40\% de las respuestas en mujeres; y $27.7 \%$ de las respuestas en hombres). El segundo lugar más mencionado por las mujeres es el transporte público (23.7\%), mientras que para los hombres el segundo lugar más mencionado son los ámbitos laborales (11.1\%). Posteriormente, se procedió a analizar las respuestas a la percepción del acoso sexual de los participantes como variable independiente, con respecto a los niveles de sexismo hostil como variable dependiente por un lado $\left(F_{(3,378)}=30.613\right.$; $p<.001$ Eta $\left.^{2}=.304\right)$ y considerando a al sexismo benevolente como variable dependiente por otro $\left(F_{(3,386)}\right.$ $\left.=37.229 ; p<.001 ; \mathrm{Eta}^{2}=.316\right)$. En la Tabla 1 se presentan los puntajes medios de ambos análisis de la varianza.

Asimismo, se estudiaron las percepciones sobre el acoso sexual de los participantes como variable independiente con respecto a los niveles de ideología del rol de género como variable dependiente $\left(F_{(3,381)}=36.540 ; p\right.$ $<.001 ; \mathrm{Eta}^{2}=.327$ ) (Tabla 2).
Tabla 1. Percepción de acoso sexual según el sexismo ambivalente

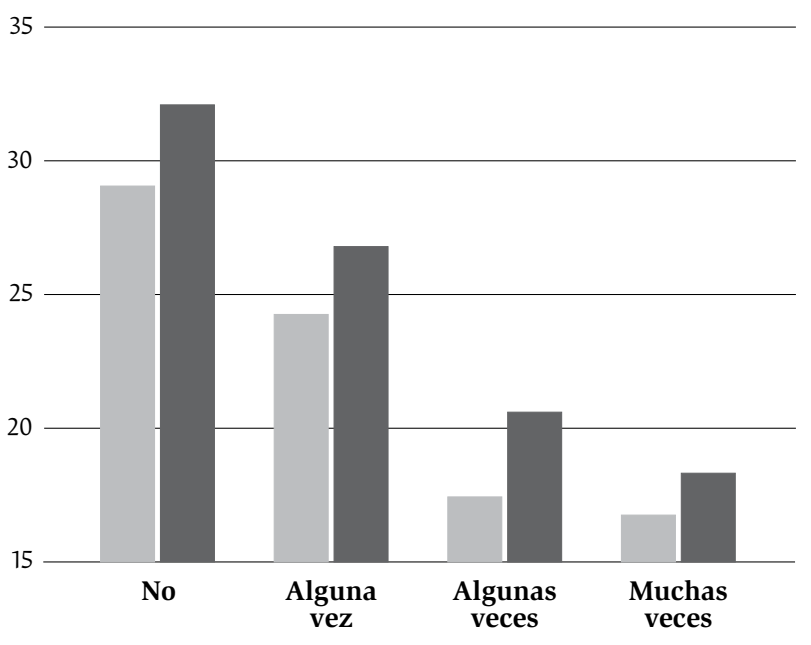

Sexismo Hostil Sexismo Benevolente

Tabla 2. Percepción de acoso sexual según ideología del rol de género

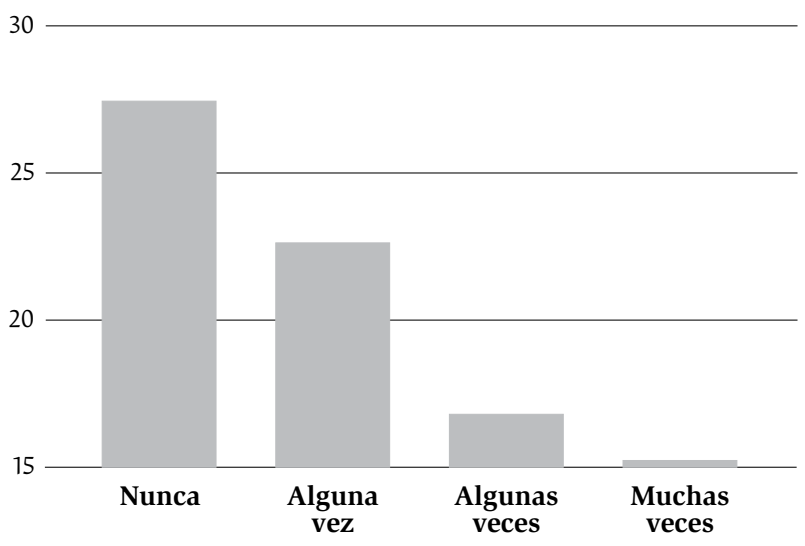

Como puede observarse en la Tabla 2, la ideología del rol de género es una variable que discrimina grupos de forma significativa según las diferentes percepciones del acoso sexual, encontrando que quienes respondieron que "Nunca" percibieron una situación de acoso sexual, poseen los mayores niveles de ideología del rol de género en la muestra, mientras que los que indicaron que percibieron "Muchas veces" el fenómeno, obtuvieron los puntajes más bajos en esta escala.

\section{Discusión}

Uno de los principales resultados hallados en este estudio es que un $35.7 \%$ de los participantes declararon no haber sufrido ni conocer a nadie que haya sufrido situaciones de acoso sexual. Los restantes participantes, oscilan entre $26.5 \%$ "alguna vez"; $22.7 \%$ "algunas veces"; $15.2 \%$ respondió "muchas veces", disminuyendo consi- 
derablemente los porcentajes. También sorprende que sólo un $15.2 \%$ declare haber percibido acoso sexual "muchas veces". Es posible considerar que actualmente diferentes comportamientos sexistas siguen siendo percibidos como naturales y no como formas de acoso sexual. En este sentido, el estudio sobre la percepción del acoso en el ámbito callejero en la Ciudad Autónoma de Buenos Aires (UAI, 2018) deja ver que el $85 \%$ de las mujeres encuestadas sufrió alguna vez una situación de acoso sexual callejero. En el caso de los varones participantes, el $62 \%$ "cree que a las mujeres les gustan los piropos callejeros” (UAI, 2018). Estos datos parecen demostrar que existe un sesgo de género al momento de percibir el fenómeno y que los hombres tienen mayor propensión a no percibir el acoso sexual, estadísticas que concuerdan con los resultados de la presente investigación. Al comparar la percepción de acoso entre hombres y mujeres, observamos que los hombres perciben significativamente menos situaciones de acoso sexual que las mujeres. Los resultados también permiten observar que, aunque las mujeres tienen una mayor percepción del acoso sexual que los hombres, es probable que tanto los hombres como las mujeres que tienen una menor percepción del acoso sexual sean más propensos a albergar actitudes de hostilidad y benevolencia hacia las mujeres. De hecho, los niveles de sexismo benevolente y de sexismo hostil son factores importantes en la identificación del acoso sexual; estos hallazgos son consistentes con investigaciones previas que encontraron que la hostilidad hacia las mujeres juega un papel importante en la identificación de las actitudes de acoso en los hombres y mujeres (Fiske, 2018; Glick \& Fiske, 1995; Pryor et al., 1995; Russell \& Oswald, 2016).

Con respecto a las diferencias en la percepción del acoso sexual con respecto a los niveles de sexismo ambivalente se observó que, tanto en sus formas hostiles como benevolentes, los mayores niveles de prejuicio sexista se observaron en los participantes que indicaron "Nunca" haber percibido una situación de acoso sexual. Los participantes no sexistas o con un menor nivel de sexismo predijeron una mayor percepción del acoso sexual, mientras que los participantes con un nivel medio de sexismo hostil percibieron el acoso sexual en niveles bajos; de forma directamente proporcional, los participantes con altos niveles de sexismo hostil mostraron resultados muy significativos de percepción limitada o no tuvieron percepción sobre situaciones de acoso. El sexismo benevolente se asoció positivamente con la falta de percepción del acoso sexual, siendo también un predictor significativo de este tipo de comportamientos.

En cuanto a la ideología del rol de género, al igual que con el prejuicio sexista, a mayores niveles de ideología del rol de género desigual, menor percepción del acoso sexual. La hipótesis de que tanto los hombres como las mujeres que apoyan una ideología de los roles de género tradicionales no perciben el acoso sexual fue respaldada por los resultados de forma significativa. Este aspecto podría deberse a que los roles de género están intrínsecamente relacionados con el prejuicio sexista tanto en su forma sutil como benevolente, en hombres y en mujeres. Los resultados evidencian que un alto nivel de prejuicio sexista es predictivo de baja o nula percepción al acoso. El sexismo benevolente, por ejemplo, connota actitudes subjetivamente positivas hacia las mujeres y tales actitudes están arraigadas en las diferencias de poder que promueven y sustentan prejuicios sobre los roles de género (Fiske, 2018; Gaertner \& Dovidio, 2014; Glick \& Fiske, 1996). Parece lógico que los individuos que respaldan la benevolencia hacia las mujeres sean más propensos a condenar comportamientos como el acoso, aunque tienden a respaldar las actitudes y comportamientos tradicionales de los roles de género y, finalmente, se vuelvan menos tolerantes con aquellos que van más allá de los límites de su propia concepción del rol de género (Russell \& Oswald, 2016).

Debido a que los resultados de la investigación muestran que hombres y mujeres comparten características en cuanto a la nula o baja percepción del acoso, y que esto se debe al alto nivel de sexismo hostil y sexismo benevolente, futuras investigaciones podrían desarrollarse en torno a la hostilidad que las mujeres adoptan hacia su propio grupo. Si el sostenimiento de una ideología de los roles de género juega un papel importante en la identificación de las actitudes de acoso, futuros estudios podrían analizar en qué medida el nivel de apoyo y reconocimiento a los roles de género por parte de las mujeres, aumenta su nivel de tolerancia y falta de percepción hacia el acoso sexual que los hombres cometen hacia su propio grupo.

Los aportes de este estudio podrían ayudar a desarrollar estrategias de concientización y prevención del acoso sexual, a través de la puesta en marcha de campañas de divulgación acerca del acoso sexual y sus dimensiones.

\section{REFERENCIAS}

Allport, G. W. (1954). The nature of prejudice. Reading, MA: Addison-Wesley.

Amnistía Internacional. (2017). Informe 2016-2017. La situación de los derechos humanos en el mundo. Recuperado de https:// www.amnesty.org/download/Documents/POL1048002017SPANISH.PDF

Comisión Económica para América Latina y el Caribe [CEPAL]. (2018). Agenda 2030 y los Objetivos de Desarrollo Sostenible. Una oportunidad para América Latina y el Caribe. Recuperado de https://repositorio.cepal.org/bitstream/handle/11362/40155/ 10/S1700334_es.pdf

Corte Suprema de Justicia de la Nación, República Argentina, Oficina de Violencia Doméstica [OVD]. (2018). Informe primer trimestre. Año 2018. Recuperado de http://old.csjn.gov.ar/docus/documentos/verdoc.jsp?ID = 109844

Defensoría del Pueblo, Ciudad Autónoma de Buenos Aires. (2017). Informe Anual 2017. Recuperado de http://www.defensoria.org.ar/informe-anual/

Deaux, K., \& Martin, D. (2003). Interpersonal networks and social categories: Specifying levels of context in identity processes. Social Psychology Quarterly, 66(2), 101-117. doi:10.2307/1519842 
Diehl, C., Rees, J. \& Bohner, G. (2016). Predicting Sexual Harassment from Hostile Sexism and Short-Term Mating Orientation: Relative Strength of Predictors Depends on Situational Priming of Power Versus Sex. Violence Against Women, 24(2), 1-21. doi: 10.1177/1077801216678092

Durante, F. \& Fiske, S. T. (2017). How social-class stereotypes maintain inequality. Current Opinion in Psychology, 18, 4348. doi:10.1016/j.copsyc.2017.07.033

Eagly, A. H. (1987). Sex differences in social behavior: A socialrole interpretation. Hillsdale, NJ: Lawrence Erlbaum Associates, Inc. doi:10.2307/2073813

Eagly, A. H, \& Karau, S. J. (2002). Role Congruity Theory of Prejudice Toward Female Leaders. Psychological Review, 109(3), 573-598. doi:10.1037/0033-295x.109.3.573

Eagly, A. H., \& Mladinic, A. (1993). Are people prejudiced against women? Some answers from research on attitudes, gender stereotypes, and judgments of competence. En W. Stroebe \& M. Hewstone (Eds.), European review of social psychology (pp. 1-35). New York, NY, US: Wiley.

Eagly, A. H., \& Wood, W. (1982). Inferred sex differences in status as a determinant of gender stereotypes about social influence. Journal of Personality and Social Psychology, 43(5), 915-928. doi: 10.1037/0022-3514.43.5.915

Eagly, A. H., Wood, W., \& Diekman, A. B. (2000). Social role theory of sex differences and similarities: A current appraisal. En T. Eckes y H. M. Trautner (Eds.), The developmental social psychology of gender (pp. 123-174). Mahwah, NJ: Erlbaum.

Escartí, A., Musitu, G. \& Gracia, M. D. (1988). Estereotipos de género y roles sexuales. En J. Fernández (Ed.), Nuevas perspectivas en el desarrollo del sexo y el género (pp. 205-225). Madrid: Pirámide.

Etchezahar, E. (2014). La construcción social del género desde la perspectiva de la Teoría de la Identidad Social. Ciencia, Docencia y Tecnología, 25(49), 128-142. Recuperado de http:// www.redalyc.org/pdf/145/14532635005.pdf

European Union Agency for Fundamental Rights [FRA]. (2014). Violence against women: An EU-wide survey (Main results). Luxembourg: Publications Office of the European Union. Recuperado de http://fra.europa.eu/sites/default/files/fra_uploads/ fra-2014-vaw-survey-main-results-apr14_en.pdf

Expósito, F. \& Moya, M. (2005). Violencia de género. En F. Expósito, y M. Moya (Eds.), Aplicando la psicología social (pp. 208216). Madrid, ES: Pirámide.

Fiske, S. T. (2018). Social Cognition. Selected Works of Susan Fiske. London, UK: Routledge.

Fiske, S. T., \& Glick, P. (1995). Ambivalence and stereotypes cause sexual harassment: A theory with implications for organizational change. Journal of Social Issues, 51(1), 97-115. doi: 10.1111/j.1540-4560.1995.tb01311.x

Gaertner, S. L. \& Dovidio, J. F. (2014). Reducing Intergroup Bias. The common Ingroup Identity Model. New York, NY: Psychology Press.

Galdi, S., Mass, A. \& Cadinu, M. (2013). Objectifying Media: Their Effect on Gender Role Norms and Sexual Harassment of Women. Psychology of Women Quarterly, 38(3), 398-413. doi: $10.1177 / 0361684313515185$

Geis, F. L. (1993). Self - fulfilling prophecies: a social psychological view of gender. En A. E. Beall y R. J. Sternberg (Eds.), The psychological of gender (pp. 9-54). Nueva York, NY: Guilford Press.
Glick, P., \& Fiske, S. T. (1996). The Ambivalent Sexism Inventory: Differentiating hostile and benevolent sexism. Journal of Personality and Social Psychology, 70, 491-512. doi: 10.1037/00223514.70.3.491

Glick, P., \& Fiske, S. T. (2001). An ambivalent alliance: Hostile and benevolent sexism as complementary justifications for gender inequality. American Psychologist, 56, 109-118. doi: 10.1037/0003-066x.56.2.109

Glick, P., \& Hilt, L., (2000). Combative children to ambivalent adults: The development of gender prejudice. En T. Eckes, y $\mathrm{H}$. Trautner (Eds.), The developmental social psychology of gender (pp. 243-272). Mahwah, NJ: Lawrence Erlbaum Associates.

Guedes, A., García-Moreno, C. \& Bott, S. (2014). Violencia contra las mujeres en Latinoamérica y el Caribe. Foreign Affairs Latinoamérica, 14(1), 41-48. Recuperado de: www.fal.itam.mx

Holland, J., Rabelo, V., Gustafson, A., Seabrook, R. \& Cortina, L. (2016). Sexual Harassment against men: examining the roles of feminist activism, sexuality, and organizational context. Psychology of Men y Masculinity, 17(1), 17-29. doi: 10.1037/ a0039151

Instituto Nacional de Estadística y Censos [INDEC]. (2018). Registro Único de Casos de Violencia contra las Mujeres (RUCVM). Resultados estadísticos 2013-2017. Recuperado de https://www. indec.gob.ar/uploads/informesdeprensa/rucvm_03_18.pdf

Instituto Nacional de las Mujeres [INAM]. (2017). Línea 144. Informe Estadístico de Casos, Tercer Trimestre 2017. Recuperado de https://www.argentina.gob.ar/sites/default/files/144infor metercertrimestre2017.pdf

Jackman, M. R. (1999). Gender, violence, and harassment. En J. Saltzman Chafetz (Ed.), Handbook of the sociology of gender (pp. 275-317). New York, NY: Springer.

Ley 26.485. Ley de protección Integral a las Mujeres. Boletín Oficial de la República Argentina. Ciudad Autónoma de Buenos Aires, Argentina, 11 de marzo de 2009. Recuperado de https://www.oas.org/dil/esp/Ley_de_Proteccion_Integral_ de_Mujeres_Argentina.pdf

McCord, M. A., Joseph, D. L., Dhanani, L. Y., \& Beus, J. M. (2018). A meta-analysis of sex and race differences in perceived workplace mistreatment. Journal of Applied Psychology, 103(2), 137-163. doi.org/10.1037/apl0000250

Moyer, R., \& Nath, A. (1998). Some effects of brief training interventions on perceptions of sexual harassment. Journal of Applied Social Psychology, 28(4), 333-356. doi: 10.1111/j.1559-1816.1998. tb01709.x

Organización de los Estados Americanos [OEA] (1994). Convención Interamericana para Prevenir, Sancionar y Erradicar la Violencia contra la Mujer. Convención de Belém do Pará. Recuperado de http://www.oas.org/es/mesecvi/docs/BelemDoPara-ESPANOL.pdf

Organización Internacional del Trabajo [OIT]. (2013). Acoso sexual en el trabajo y masculinidad: Exploración con hombres de la población general. Centroamérica y República Dominicana. Exploración con hombres de la población general. Recuperado de http://www.ilo.org/sanjose/sala-de-prensa/WCMS_205747/ lang--es/index.html 
Organización Internacional del Trabajo [OIT]. (2017). Acabar con la violencia y el acoso contra las mujeres y los hombres en el mundo del trabajo. Conferencia Internacional del Trabajo, $107^{a}$ reunión. Recuperado de https://www.ilo.org/wcmsp5/ groups/public/---ed_norm/---relconf/documents/meetingdocument/wcms_554100.pdf

Organización de las Naciones Unidas [ONU]. (1979). Convención sobre la Eliminación de todas las formas de Discriminación contra la Mujer (CEDAW). Recuperado de https://www.unicef. org/panama/spanish/MujeresCo_web.pdf

Organización de las Naciones Unidas [ONU]. (1995). Declaración y Plataforma de Acción de Beijing. Convención de Beijing. Recuperado de http://www.un.org/womenwatch/daw/beijing/ pdf/Beijing\%20full\%20report\%20S.pdf

Organización de las Naciones Unidas [ONU]. (2015). Agenda 2030 para el Desarrollo Sostenible. Recuperado de https:// www.un.org/sustainabledevelopment/es/gender-equality/

Organización de las Naciones Unidas [ONU]. (2017). Informe de los Objetivos de Desarrollo Sostenible. Recuperado de http:// ggim.un.org/documents/TheSustainableDevelopmentGoalsReport2017_Spanish.pdf

Organización Mundial de la Salud [OMS]. (2013). Comprender y abordar la violencia contra las mujeres. Consecuencias para la salud. Recuperado de http://www.who.int/reproductivehealth/ publications/violence/rhr12_43/es/

Organización para la Cooperación y el Desarrollo Económicos [OECD]. (2014). Reporte regional Índice de Instituciones Sociales y Género (SIGI). Recuperado de https://www.oecd.org/ dev/development-gender/Mini-Brochure_SIGI_AmericaLatinayelCaribe-web.pdf

Pratto, E., Sidanius, J., \& Stallworth, L. M. (1993). Sexual selection and the sexual and ethnic basis of social hierarchy. En L. Ellis (Eds.), Social stratification and socioeconomic inequality (Vol. 1, pp. 111-137). Westport, CT: Praeger Publishers.

Pryor, J.B. (1987). Sexual harassment proclivities in men. Sex Roles, 17(5-6), 269-290. doi: 10.1007/BF00288453

Pryor, J. B., Giedd, J. L., \& Williams, K. B. (1995). A social psychological model for predicting sexual harassment. Journal of Social Issues, 51, 69-84. doi: 10.1111/j.1540-4560.1995.tb01309.x
Pryor, J. B., \& Whalen, N. J. (1997). A typology of sexual harassment. Characteristics of harassers and the social circumstances under which sexual harassment occurs. En W. O'Donohue (Ed.), Sexual harassment: Theory, research and treatment (pp. 129-151). Boston, MA: Allyn and Bacon.

Ramírez, C. (2016). Las violencias contra las mujeres: alcances y consecuencias. Recuperado del sitio del Diccionario Enciclopédico de la Legislación Sanitaria Argentina, Ministerio de Salud de la Nación: http://www.salud.gob.ar/dels/entradas/ las-violencias-contra-las-mujeres-alcances-y-consecuencias

Reyes Aguinaga, H. (1998). Relaciones de género y machismo. Entre el estereotipo y la realidad. Iconos, 5, 84-94. doi: https:// doi.org/10.17141/iconos.5.1998.631

Russell, B. L., \& Oswald, D. (2016). When Sexism Cuts Both Ways: Predictors of Tolerance of Sexual Harassment of Men. Men and Masculinities, 19(5), 524-544. doi: 10.1177/1097184X15602745

Russell, B. L., \& Trigg, K. Y. (2004). Tolerance of Sexual Harassment: An Examination of Gender Differences, Ambivalent Sexism, Social Dominance, and Gender Roles. Sex Roles, 50(7-8), 565-573. doi: 10.1023/b:sers.0000023075.32252.f

Stockdale, M. S. (1998). The Direct and Moderating Influences of Sexual-Harassment Pervasiveness, Coping Strategies, and Gender on Work-Related Outcomes. Psychology of Women Quarterly, 22(4), 521-535. doi:10.1111/j.1471-6402.1998.tb00175.x

Stockdale, M. S., Gandolfo, C., Schneider, R. W. \& Cao, F. (2004). Perceptions of the Sexual Harassment of Men. Psychology of Men and Masculinity, 5(2), 158-167. doi:10.1037/1524-9220.5.2.158

Ungaretti, J. \& Etchezahar, E. (2013). Gender Role Ideology according to Sex, Acceptance of Women's Rights and Gay Marriage. International Journal of Humanities and Social Science, 3(15), 40-45. Recuperado de http://www.ijhssnet.com/ journals/Vol_3_No_15_August_2013/5.pdf

Universidad Abierta Interamericana [UAI]. (2018). Encuesta sobre Acoso Callejero. Recuperado de: https://noticias.uai.edu. ar/facultades/psicolog\%C3\%ADa-y-relaciones-humanas/encuesta-sobre-acoso-callejero/ 